\title{
An Approach to Collaborative Scheduling Through Group Decision Support
}

\author{
Ana Almeida, and Goreti Marreiros \\ Knowledge Engineering and Decision Support Group, Institute of Engineering, Polytechnic of Porto \\ Rua Dr. Antonio Bernardino de Almeida, 4200-072 Porto, Portugal \\ E-mail: \{ana, goreti\}@dei.isep.ipp.pt \\ [Received September 23, 2005; accepted December 19, 2005]
}

The model we present supporting collaborative scheduling in complex dynamic manufacturing environments, considers the interaction between an agentbased scheduling module (ASM) and a group decision support module (GDSM). The ASM outputs a set of candidate scheduling solutions, each generated based on specific criteria and/or by a particular method. Scheduling is a multicriteria decision problem in practice where different schedulers may agree on key objectives but differ greatly on their relative importance in any given situation. Interaction among scheduling actors is supported by the GDSM selecting a scheduling solution.

Keywords: group decision support, collaborative and cooperative scheduling

\section{Introduction}

A major fact in an organization's competitiveness and success is efficient production management. Client satisfaction means delivering low cost high quality products or services on time.

Production scheduling may become complex when dealing with dynamic environments characterized by high variation factors. This scenario becomes more problematic when the variables behaviour is not the same as planned, raising the strong possibility of reformulating existing planning and changing current scheduling to adapt to emerging new needs.

Scheduling is an area in which decision support systems (DSSs) are more advantageous due to the complexity and dynamic nature of the environment and the different actors involved.

Group decision-making plays an important role in organizations. The new economy demands that decisions be made quickly without adversely affecting the quality of the decision-making process or its results.

With the objective of making better decisions, more frequently decisions are taken for groups of individuals representing different organizational perspectives.

Problems faced by organizations, particularly in scheduling, involve the evaluation and selection of an al- ternative among a set of alternatives. These decisions are not trivial because usually involve multiple and conflicting criteria.

Despite the great variety of DSSs, most are individual tools developed to help a particular user involved in a specific decision process. In management most decisions made require prior consultation with more than one person to discuss and argue about alternatives. Group decision support systems (GDSSs) thus have emerged as a vital important area in the management domain, providing collaborative frameworks for decision support.

Generally, the present business environment features the use of groups, which work in distributed environments and deal with uncertainty, ambiguous problem definitions, and rapidly changing information.

We focus on interaction between scheduling actors through the integration of different types of knowledge in a global view of the system and potential synergy associated with the collaborative activity of actors taking in account multiple criteria that potentially, can improve scheduling. Given this factor the option for a collaborative model using the GDSSs concept plays an important role. Practical advantages involve better performance among managers responsible for production planning and control and consequently increased efficiency and productivity of industrial systems.

This paper is organized as follows: Section 2 provides a general approach to scheduling, focusing on collaborative scheduling. Section 3 introduces GDSSs. Section 4 discusses the model we propose for supporting decisions in collaborative scheduling and its features. Section 5 presents conclusions, perspectives and ideas for projected work.

\section{Collaborative Scheduling}

Scheduling involves solving two problems - allocating resources to tasks and determining when task are to be done [1]. Real-life scheduling problems tend to be so complex, that usually are NP-hard [2] making optimum solutions generally intangible.

Based on how production scheduling problems have been conventionally tackled, we may conclude that a great disparity exists between how scheduling systems solve 
problems and how human solve them. While automatic scheduling requires the complete specification of goals and scenarios before beginning problem solving, persons progressively learn by scenario and change goals. Automatic scheduling quantitatively evaluates plans while persons evaluate them subjectively. While automatic systems focus on one solution at a time, persons compare options and alternatives before choosing a route [3]. Evaluation criteria vary and are often contradictory, arising from diverse manufacturing perspectives involved in scheduling. Actual industrial environments are often geographically dispersed and unplanned scheduling environment events occur frequently, requiring that scheduling decisions be made constantly. This scheduling is referred to as distributed dynamic scheduling (DDS) and incorporates online information in real time enabling the adjustment of data and schedules as needed. The need thus exists for a diverse range of technical capabilities, usually representing different manufacturing perspectives working together, sharing knowledge through collaboration to rich a global scheduling solution.

The determination of feasible and mutually acceptable schedules is a major challenge [4]. Automated scheduling, whatever its nature whether heuristic or rulebased, may not produce realistic schedules in environments where contextual information is represented inadequately. Where objectives are complex and unstated and situations are dynamic and uncertain, domain experts address these issues [5-8] bringing to scheduling their inductive and pattern recognition abilities.

This evidence the need to create collaborative scheduling in which users and scheduling engines collaborate in plans generation, identifying alternative candidates, and selection one of them, thus bringing out the best of two worlds. Collaboration may thus provide a very powerful approach to multiattribute, multicriteria, decision support in complex manufacturing environments.

Collaborative scheduling occurs where different organizational units coordinate individual activities for joint benefit. Participants deal with multiattribute, multiparty, multicriteria decision-making and negotiation - this environment is characterized by distributed, uncertain, and conflicting contextual information. Typically, this complexity is neither adequately modelled mathematically nor sufficiently captured in information databases [9] making human schedulers crucial to collaborative scheduling.

An example of work on distributed, cooperative, or collaborative scheduling is that developed by Kawamura et al. [10], a distributed cooperative scheduling system in which several scheduling agents negotiate among themselves to adjust schedules among busy departments. An alternative approach, by Murthy et al. [11], involves autonomous agents working together to produce a set of alternative candidates and a human scheduler making the final decision interacting with other agents. Chang et al. [12] developed a collaborative scheduling system for coordinating work schedules in the construction industry, introducing a dependency intelligence list and a mechanism for resolving concurrency problems, as collaborative fea- tures. Cooperation and collaboration are interpreted as coordinated negotiations triggered by user intervention.

Our approach to collaborative scheduling considers the integration of multiple problem-solving approaches to produce a set of solutions to a scheduling problem and the interaction between group decision-makers using different evaluation criteria to arrive at a single solution.

This collaboration involves interaction between a group of persons from several departments who are decisionmakers representing different manufacturing perspectives. In practice, different schedulers may agree to key objectives but differ greatly about their relative importance in a given situation. It thus requires substantial effort to define solutions that are feasible and efficient and encompass multiple perspectives. Our methodology is applicable to scheduling problems having no dominant solution.

\section{Group Decision Support}

Manufacturing environments are generally highly uncertain and dynamic, making scheduling an ongoing process of responding to unexpected and evolving circumstances for which optimal solutions usually cannot be found.

According to Bedworth [13] "... common sense is the best way of scheduling when scenery is complex". What seems really useful is a tool for supporting decisions to help operators achieve and contribute to good scheduling. This requires a collaborative framework to integrate multicriteria decisions arising from different actors involved in manufacturing.

The benefits of group work are several, and in our opinion have great application to manufacturing environments:

- Groups are better than individuals at understanding problems.

- People are more responsible for decisions in which they participate, which mean less likely to resist implementation.

- A group is better than an individual at detecting flaws in proposed ideas.

- A group has more knowledge than any single member.

- Synergy may develop making groups effectiveness greater than that which could have been produced individually. Working in a group may stimulate members and, consequently, decisions making; $\equiv$

- Participants' differing knowledge and processing skills allow for results that could not have been achieved individually.

If there are advantages associated with group work, there are also disadvantages: 
- Time-consuming aspect - $\equiv \mathrm{p}$ work is slow, only group member can speak at time, and the tendency for repetition of what has already been said is high.

- High cost - many hours of participation, travel time, travel expenses, etc are involved.

- Improper use of group dynamics - domination of time, or opinion by one or a few members, and fear of speaking in front of others may disturb the process.

- The tendency exists to rely on a few core members most of the time.

- Task analysis tends to be incomplete and compromise solutions tend to be of poor quality.

GDSS reduces loss associated with group work and helps to improve gain [14].

The term GDSS [15-17] emerged effectively at the beginning of the 80's. According to Huber [18], a GDSS consists of a set of software, hardware, languages components and procedures that support a group of people engaged in a decision-related meeting. A more recent definition by Nunamaker et al [19] states that GDSSs are an interactive computer-based environment that supports concerted, coordinated team effort toward the completion of joint tasks.

Some advantages of using GDSS are:

- Equal and anonymous opportunities to contribute ideas and opinions.

- Parallel communication between group members (who could interact simultaneously).

- Helping the meeting facilitator in schedule management.

- Eliminating domination of certain members in the meeting.

- Automatically organizational memory, through automatic recording of meeting information.

- Finding common and dissenting preferences among group members.

Using GDSSs enables groups to integrate the knowledge of all members into better decision-making.

DeSanctis and Gallupe [20] proposed a classification for GDSS based on matrix time/place:

In the 80s, most GDSS research focused on the synchronous/same-place dimension, in which several decision rooms were configured. With the proliferation of the Internet, GDSS research has focused on different-time/different-place dimension. Several Webbased GDSSs have been developed [21-23], and others, e.g., GroupSystems, initially developed to support the configuration of decision room, are now able to support remote decision-making.

As mentioned in the introduction, scheduling involves the evaluation and selection of one among alternatives.
Table 1. GDSS classification (from DeSanctis and Gallupe, 1985).

\begin{tabular}{|l|l|l|}
\hline & Synchronous & Asynchronous \\
\hline $\begin{array}{l}\text { Same } \\
\text { place }\end{array}$ & $\begin{array}{l}\text { Decision } \\
\text { Room }\end{array}$ & $\begin{array}{l}\text { Local Decision } \\
\text { Network }\end{array}$ \\
\hline $\begin{array}{l}\text { Different } \\
\text { place }\end{array}$ & Teleconference & $\begin{array}{l}\text { Remote Decision- } \\
\text { Making }\end{array}$ \\
\hline
\end{tabular}

These are not trivial decisions, because they usually involve multiple conflicting criteria. Organizations tend to be globally dispersed, e.g., in Portugal several textile industries have their administrations in Portugal, $\mathrm{b} \equiv \mathrm{O}$ duction sections in China. Agents involved in scheculing are in different countries and if it they are to discuss possible scheduling alternatives, require a support infrastructure.

We propose a model to support collaborative scheduling in complex dynamic manufacturing environments based on GDSS concept.

\section{Scheduling Model Based on GDSS}

Scheduling should provide user support in building, changing, and revising scheduling plans but not making decisions for users, who have intelligence and knowledge acquired over time that are not to be underestimated. Scheduling should be autonomous, suggesting alternatives based on predetermined or agreed-upon criteria.

Users provide intuition, notions about goals and appropriate trade-off, and refined problem resolution strategies. The computer provides skill in managing details, to assign and schedule resources and operations, and to quantitatively analyze the suggested choices.

Our proposal considers multiple scheduling objectives in a collaborative global multicriteria framework, generating scheduling alternatives by means of autonomous agents using different scheduling algorithms. Each scheduling alternative represents a solution for an objective such as accomplishment of deadlines, minimizing throughput time, maximizing profitability, product quality, and minimizing manufacturing disruptions. It provides decision support considering the negotiation process of a group of users, each with a different perception of the problem, effectively acting as a team to achieve a common unique solution. The proposed model (Fig.1) includes two modules - the ASM responsible for creating a set of scheduling solutions and the GDSM in charge of selecting a scheduling solution.

\subsection{ASM}

Agents and multiagent systems are important in AI R\&D. Agents are typically knowledge representation entities characterized by independence and autonomy. Software agents are entities that have the ability to plan and es- 


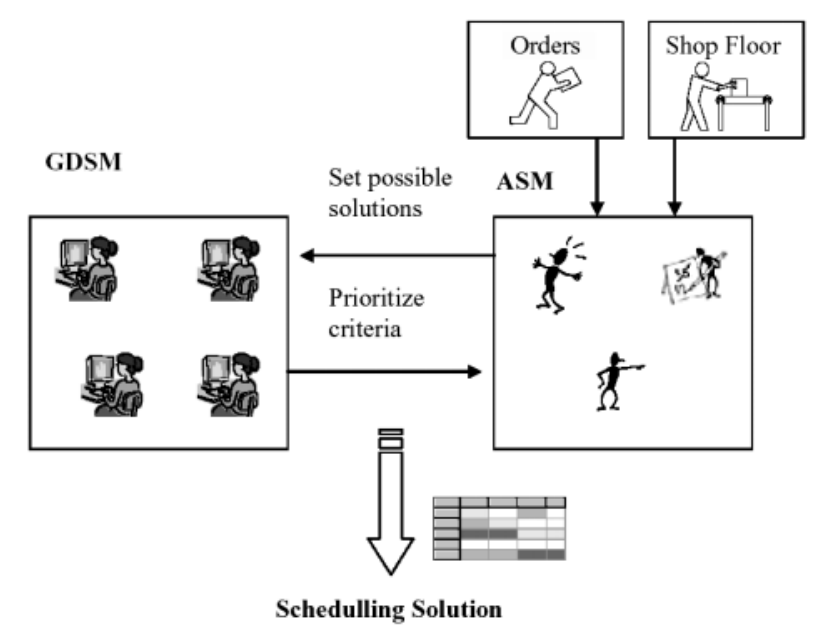

Fig. 1. Collaborative decision support framework model for scheduling.

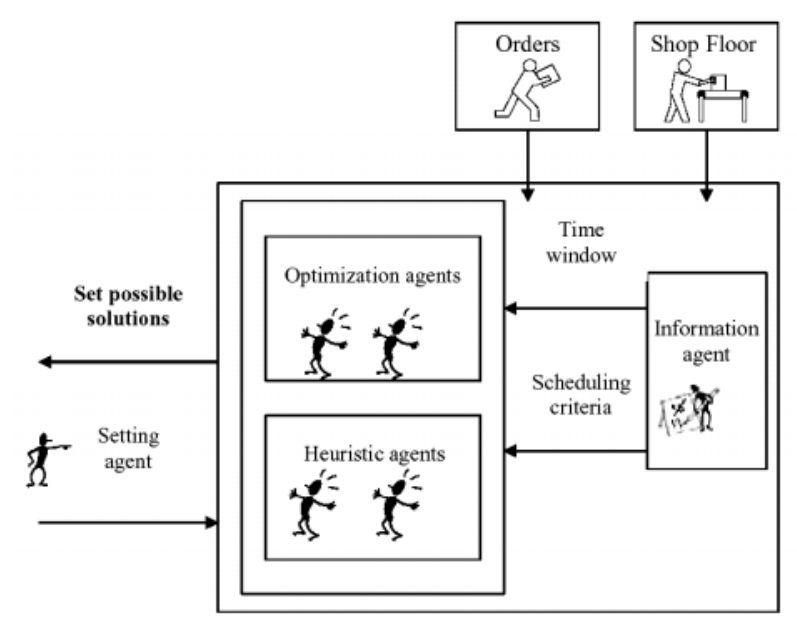

Fig. 2. ASM architecture.

tablish actions ahead of time, develop appropriated problem solving strategies, communicate, or share resources.

In our approach, agents follow events as they occur in the environment, interpreting and sharing knowledge or data.

The ASM consists of multiple problem-solving methods (called agents) working simultaneously on a common problem, so it represents any no single method or heuristic, but is rather an attempt to use multiple techniques by encapsulating individual algorithms as autonomous agents. The ASM module includes different autonomous agents - information agents, scheduling agents, and setting agents (Fig.2).

Scheduling agents are different autonomous agents, each embodying a particular scheduling algorithm as stated previously. Depending on scheduling criteria or objectives, a broad range and variety of scheduling methods exists.

We distinguish among optimization methods and heuristic algorithms. With optimization we expect an op- timal solution, while with heuristic algorithms, we expect only a good solution. Computation time generally differ significantly, usually making the difference in the choice of which to use. The literature provides a broad study on scheduling methods diversity $[24,25]$.

In our approach, the information agent, based on the type of scheduling problem, sets a time window for generating several scheduling alternatives together with some criteria. This way, only agents embodying algorithms respecting established criteria are triggered. Only alternatives generated within the time window are considered for analysis and discussion by the GDSS module.

The setting agent prioritizes criteria importance based on global preferences of GDSS members, to cover all relationships arising from different departments.

\subsection{GDSM}

One approach to tackling multicriteria decision problems involves assigning weights to different criteria, to come to a unique decision based on the assigned weights. In collaborative decision-making, which frequently involves many people, experts on different aspects of the problem, all relationships arising from different departments representing diverse manufacturing perspectives must be considered, so a set of weighted criteria seems most efficient. A manager may view profit as the most important criteria, while quality control department representative may emphasize product quality. As economic conditions change, the relative importance of different criteria may change [11], requiring users to modify weighting factors by changing the relative importance of each criterion.

Architectures that enable collaboration are useful when a single agent or person can not efficiently or otherwise do a task. They provide mechanisms enabling several users to contribute their knowledge, participating equally in selecting alternatives.

One way of enhancing collaboration between agents and persons is to produce many candidate solutions, evaluated based on multiple criteria. This enables users to gain important insights into trade-offs between multiple competing objectives. They express their preferences by weighting different criteria.

A GDSS provide a very powerful approach to multicriteria decision support and optimization in complex manufacturing environments.

The GDSM supports members of a scheduling meeting and the facilitator, who prepares the meeting and invites participants to exchange different points of view, expertise, and information to choose the "best" solution from the set of scheduling solutions proposed by the ASM. The GDSM consists of setup, management, argumentation, multicriteria, voting, and database components [26] (Fig.3)

\subsubsection{Setup Component}

The setup component is operated by the facilitator during the premeeting phase in configuration and parameter- 


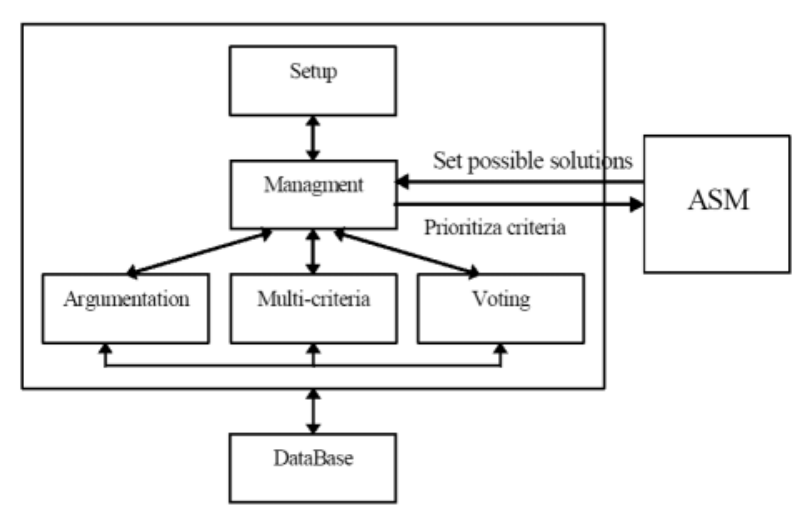

Fig. 3. GDSM architecture.

ization activities, such as the following:

- General schedule meeting configuration - specifying dates and times for the start, end, and goal of the meeting.

- Selection of participants - invitation sending, confirmation of reception, and participant replacement, if necessary. For each participant, the facilitator also defines importance (number of votes).

- Definition of decision rules - voting rules (consensus, majority, qualified majority, maximum number of voting cycles, anonymity), rules for argumentation (minimum/maximum number of arguments for each participant; arguments visible or not to all the participants), single or multiple alternative selection; visualization (or not) of the values of alternatives after normalization.

\subsubsection{Management Component}

The management supports the meeting in all its phases, sending notifications by e-mail to the facilitator or to group members and is responsible for the communications with the ASM.

\subsubsection{Multicriteria Component}

The multicriteria component is used by the facilitator to introduce a possible set of criteria, e.g., delivery time, quality, or price. Group members individually assign weights to criteria, resulting in several sets of criteria weights, one for each group member. The group consists of $k$ elements.

$$
\begin{aligned}
& W^{T}=\left(w_{1}, w_{2}, \ldots, w_{j}, \ldots, w_{k}\right) \ldots \ldots \\
& \sum_{j=1}^{n} w_{j}=1, w_{j} \geq 0 \ldots \ldots
\end{aligned}
$$

where $n$ is the number of criteria and $w_{j}$ is the weight of criterion $j$. The sum of criteria weights should equal 1 , and all criteria should have a weight greater to or equal to 0 .
No set of alternatives exists, only sets of criteria weights, and the system may take one of two different actions:

- Mathematical aggregation of individual preference, to have a unique set of criteria weights.

- Group members could use argumentation and voting tools to select a unique set of criteria weights.

The first is certainly less time-consuming, but the second is more consensual.

After the ASM module identifies the set of candidate alternatives, group members use this module to individually choose the preferred alternative, using two distinct methods both based on multiattribute value function theory with a compensatory option to rank alternatives - the Technique for Order Preference by Similarity to the Ideal Solution (TOPSIS) algorithm [27] an $\equiv$ imple additive function, such as that in equation 3 .

$$
f\left(a_{i}\right)=\sum_{j=1}^{n} W_{j} V_{j}\left(a_{j}\right) \quad \ldots \ldots . . . . . .
$$

where $a_{i}$ is alternative number $i, W_{j}$ is the weight of criterion $j$, and $V_{j}\left(a_{i}\right)$ is the value of alternative $a_{i}$ in criterion $j$.

Establishing preferences is evolutionary and, as time passes, members change the relative importance of each criterion and consequently express changes in their individual preferences. This component helps the individual scheduler agent rank several scheduling alternatives and may also help the user define criteria importance.

\subsubsection{Voting Component}

The voting component is responsible for sending vote bulletins and for publishing intermediate and final results. These activities are based on decision rules predefined by the facilitator in the premeeting phase.

\subsubsection{Argumentation Component}

After establishing individual preferences through the multicriteria component or with the help of other tools, or simply by intuition, participants are expected to defend these preferences, each participant arguing for the most interesting alternative or against the worst alternative, depending on their preferences. By expressing their arguments, participants expect to influence others' opinions and change their choices. This component structures discussions between group members.

\section{Conclusions}

Attention to the individual needs of customers is a driving force behind many changes in industry sectors, so a scheduling decision must take into account the knowledge and experience of participants with different points of view, allowing the consideration of broad issues of the 
company rather than focusing on scheduling tasks for a single process.

By functioning in a collaborative framework, our system relieves scheduler responsible of mundane computational tasks and enables them to focus on enterprise and decision-making objectives. For generating multiple solutions and facilitating cooperation with the scheduler, our system is implemented using an agent-based collaborative architecture in which schedules are shared with scheduling responsible for different stages of production and with customer service representatives and serve as a basis for negotiating a set of solutions to reach the best alternative for the enterprise.

Our proposed approach addresses many of the limitations of existing job-shop scheduling identified in [5] by providing a decision support collaborative framework for scheduling. It enables scheduling solutions to be evaluated by experts with different points of view incorporating their knowledge in the system.

Improving schedule quality has obvious impact on profitability. Just as important, however, are opportunities to change business processes that our system affords by showing multiple good alternatives, some of which may violate constraints. These solutions suggest good opportunities to look for alternative means of production and suggest when it would be profitable to negotiate to change customer requirements or business policies. If deadlines imposed by a particular costumer, for example, are always short and can never be met, it may indicate that it would be better to negotiate longer deadlines.

We expect significant savings and improved customer satisfaction with our proposal - positive results arising from improved schedule quality and improvements in the business process that our collaborative decision support approach fosters.

\section{References:}

[1] K. R. Baker, "Introduction to Sequencing and Scheduling," John Wiley \& Sons, (New York, USA), 1974.

[2] E. L. Lawler, J. K. Lenstra, A. H. G. Rinnooy Kan, and D. B. Shmoys, "Sequencing and scheduling: algorithms and complexity," in Handbooks Operations Research and Management Science, Vol.4, 1993.

[3] J. Marinho, "SCALINTEL: A DSS Prototype with Scalable Intelligence," Proceedings of the 1st International Conference On Knowledge Engineering and Decision Support (CKEDS'04), 2004.

[4] R. Kowalczyk, V. Phiong, S. Dunstall, and B. Owens, "Towards supporting collaborative scheduling in adaptive supply networks with negotiation agents," Proc. Theory and Applications of eNegotiations, Poznan, Poland, 2004

[5] K. N. McKay, F. R. Safayeni, and J. A. Buzacott, "Job-Shop Scheduling Theory: What is relevant?, Interfaces, Vol.18, pp. 8490, 1988

[6] P. G. Higgins, and A. Wirth, "Interactive Job-Shop Scheduling: How to Combine Operations Research Heuristics With Human Abilities," 6th International Conference on Manufacturing, Melbourne, Australia, pp. 293-302, 1995.

[7] V. C. S. Wiers, "Human-Computer Interaction in Production Scheduling: Analysis and Design of Decision Support Systems for Production Scheduling Tasks," Ph.D. Thesis, Eindhoven University of Technology, Holland, 1997

[8] A. Almeida, C. Ramos, and C. Sílvio, "Toward Dynamic Scheduling of Manufacturing," The International Journal for Manufacturing Science \& Production, Freund Publishing House, Ltd., 2002.

[9] K. Nezamirad, P. G. Higgins, and S. Dunstall, "Modelling Human Aspects of Collaborative Scheduling," 9th International Conference on Human Aspects of Advanced Manufacturing Agility and Hybrid Automation (HAAMAHA'04), Galway, Ireland, 2004.

[10] T. Kawamura, N. Kase, D. Araki, and A. Osuga, "Development of a Distributed Cooperative Scheduling System Based on Negotiations between Scheduling Agents," Systems and Computers in Japan, Vol.31, No.1, 2000

[11] S. Murthy, R. Akkiraju, J. Raclin, and F. Wu, "Agent-based cooperative scheduling," AAAI97 Workshop on Constraints and Agents, pp. 112-117, 1997.

[12] W. T. Chan, D. K. H. Chua, and X. Lang, "Collaborative Scheduling over the Internet," Computer-Aided Civil and Infrastructure Engineering, Vol.14, pp. 15-24, 1999.

[13] D. D. Bedworth, and J. E. Bailey, "Integrated Production Control Systems," Management, Analysis, Design 2/E, John Willey \& Sons, 1987.

[14] C. W. Holsapple, and A. B. Whinston, "Decision support systems: a knowledge-based approach," Thomson Learning, inc, 2001.

[15] G. P. Huber, "Group decision support systems as aids in the use of structured group management techniques," Proc. of second international conference on decision support systems, San Francisco, 1982, In C. W. Holsapple, and A. B. Whinston, Decision support systems: a knowledge-based approach, Thomson Learning Inc., 2001.

[16] D. Kull, "Group Decisions: Can computer Help," Computer Decisions Vol.14, No.5, 1982, In C. W. Holsapple, and A. B. Whinston, Decision support systems: a knowledge-based approach, Thomson Learning, Inc, 2001.

[17] L. F. Lewis, "Facilitator: A microcomputer decision support systems for small groups," Ph. D. dissertation, University of Louisville, 1982, In C. W. Holsapple, and A. B. Whinston, Decision support systems: a knowledge-based approach, Thomson Learning, Inc, 2001

[18] G. P Huber, "Issues in the design of group decision support systems," Mis Quarteley, Vol.3, No.8, 1984.

[19] J. F. Nunamaker et al., "Lessons from a dozen years of group support systems research: A discussion of lab and field findings," Journal of Management Information Systems, Vol.13, No.3, 1997.

[20] G. DeSanctis, and R. B. Gallupe, "Group Decision Support Systems: A New Frontier," Database, 16/1, pp. 3-10, 1985.

[21] G. Marreiros, J. P. Sousa, and C. Ramos, "WebMeeting - a group decision support system for multi-criteria decision problems," International Conference on Knowledge Engineering and Decision Support ICKEDS04, 2004.

[22] P. Shim, M. Warkentin, J. F. Courtney, D. J. Power, and R. Sharda, "Past, present, and future of decision support technology, Journal of Decision Support Systems," Vol.33, No.2, pp. 111-126, 2002.

[23] A. Dennis et al., "Using Internet to implement support for distributed decision Making," In P. Humphreys, L. Bannon, A. McCosh, P. Migliarese, and J-C Pomerol (Eds.), Implementing Systems for Supporting Management Decision: Concepts, methods, and experiences, Chapman \& Hall, UK, pp. 139-159, 1996.

[24] T. E. Morton, and D. W. Pentico, "Heuristic Scheduling Systems," John Willey \& Sons, Inc., 2003.

[25] A. Almeida, "Analysis and Development of Mechanisms and Algorithms to Support Product Oriented Scheduling," Doctoral Thesis on Production Engineering, University of Minho, Portugal, 2002.

[26] $\equiv$ rreiros, "Um Sistema de Apoio à Tomada de Decisão em , Dissertação de mestrado," Faculdade de Engenharia da Universidade do Porto, Outubro de 2002, Nobel Laureate, His book, Publisher, Location, Year.

[27] C. L. Hwang, and K. Yoon, "Multiple attribute decision making," Lectures Notes in Economics and mathematical Systems 186, Springer-Verlag Berlin/Heidelberg/New York, 1981. 


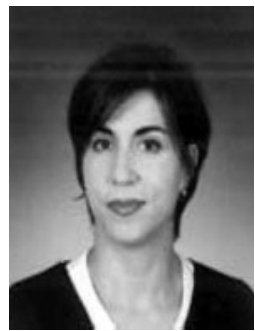

Name:

Ana Almeida

\section{Affiliation:}

Knowledge Engineering and Decision Support Group, Institute of Engineering, Polytechnic of Porto

Address:

Rua Dr. Antonio Bernardino de Almeida, 4200-072 Porto, Portugal

Brief Biographical History:

1996- Professor of the Computer Science Department at the Institute of Engineering, Polytechnic of Porto (ISEP/IPP) and researcher at the Knowledge Engineering and Decision Support Research Group (GECAD)

Main Works:

- A. Almeida, C. Ramos, and S. C. Silva, "Toward Dynamic Scheduling of Manufacturing," The International Journal for Manufacturing Science \& Production, Vol.4, No.3, pp. 169-179, Freund Publishing House Ltd., 2002.

- A. Almeida, C. Ramos, and S. C. Silva, "Product Oriented Detailed Scheduling," Computer Integrated Manufacturing, pp. 25-32, Wisla Publisher, 2003.

- A. Almeida, and C. Ramos, "A Scheduling Approach to Batch Production Envisaging the Reduction of Job Throughput Times," INES

Book, Intelligent Systems at The service of Man Kind, Vol.2, Eds. J. M. T. Machado, W. Elmenreich, and I. J. Rudas, UBooks in Augsburg, Germany, 2005.

Membership in Learned Societies:

- IASTED Technical Committee on Artificial Intelligence and Expert

Systems for the term 2005-2008

- World Enformatika Society

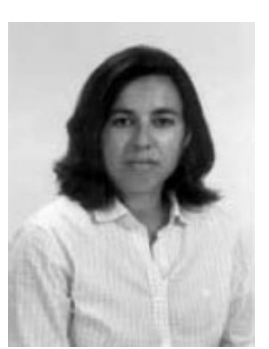

Name:

Goreti Marreiros

\section{Affiliation:}

GECAD, Knowledge Engineering and Decision Support Group, Institute of Engineering, Polytechnic of Porto

Address:

Rua Dr. Antonio Bernardino de Almeida, 4200-072 Porto, Portugal Brief Biographical History:

1995- Programmer Analyst at CSIN corporation

1998- Assistant professor of Computer Engineering at the Institute of

Engineering, Polytechnic of Porto (ISEP/IPP) and researcher at the

Knowledge Engineering and Decision Support Research Group (GECAD)

Main Works:

- G. Marreiros, C. Ramos, and J. Neves, "Modelling group decision meeting participants with an Agent-based approach," Selected for publication in an upcoming special issue of the International Journal of Engineering Intelligent Systems, 2006.

- G. Marreiros, C. Ramos, and J. Neves, "Dealing with Emotional Factors in Agent Based Ubiquitous Group Decision," Lecture Notes in Computer Science, Vol.3823, pp. 41-50, ISBN: 3-540-30803-2, Nov., 2005. 